\title{
Energy Awareness for Supercapacitors using Kalman Filter State-of-Charge Tracking
}

\author{
Andrew Nadeau ${ }^{\mathrm{a}, 1}$, Moeen Hassanalieragh ${ }^{\mathrm{a}}$, Gaurav Sharma ${ }^{\mathrm{a}}$, Tolga Soyata ${ }^{\mathrm{a}}$ \\ ${ }^{a}$ Dept. of Electrical \& Computer Engineering, University of Rochester, PO Box 270126, \\ Rochester NY 14627-0126 USA
}

\begin{abstract}
Among energy buffering alternatives, supercapacitors can provide unmatched efficiency and durability. Additionally, the direct relation between a supercapacitor's terminal voltage and stored energy can improve energy awareness. However, a simple capacitive approximation cannot adequately represent the stored energy in a supercapacitor. It is shown that the three branch equivalent circuit model provides more accurate energy awareness. This equivalent circuit uses three capacitances and associated resistances to represent the supercapacitor's internal SOC (state-of-charge). However, the SOC cannot be determined from one observation of the terminal voltage, and must be tracked over time using inexact measurements. We present: 1) a Kalman filtering solution for tracking the SOC; 2) an on-line system identification procedure to efficiently estimate the equivalent circuit's parameters; and 3) experimental validation of both parameter estimation and SOC tracking for $5 \mathrm{~F}, 10 \mathrm{~F}, 50 \mathrm{~F}$, and $350 \mathrm{~F}$ supercapacitors. Validation is done within the operating range of a solar powered application and the associated power variability due to energy harvesting. The proposed techniques are benchmarked against the simple capacitive model and prior parameter estimation techniques, and provide a $67 \%$ reduction in root-
\end{abstract}

\footnotetext{
This work was supported in part by the National Science Foundation grant CNS-1239423. A preliminary version of this paper was presented at IEEE Intl. Conf. on Acoustics Speech and Sig. Proc. (ICASSP 2014), Florence, Italy, 2014 [1].

Email addresses: andrew.nadeau@rochester.edu (Andrew Nadeau),

m.hassanalieragh@rochester.edu (Moeen Hassanalieragh), gaurav. sharma@rochester.edu

(Gaurav Sharma), tolga.soyata@rochester.edu (Tolga Soyata)

${ }^{1}$ Corresponding author: +1 315-244-7132 (phone), +1 585-273-4919, (fax)
}

Preprint submitted to Journal of Power Sources

July 10, 2015

(C) 2015. This manuscript version is made available under the Elsevier user license http://www.elsevier.com/open-access/userlicense/1.0/ 
mean-square error for predicting usable buffered energy.

Keywords: supercapacitor, three branch model, state of charge, parameter estimation, Kalman, energy awareness.

\section{Introduction}

Supercapacitors represent an emerging and rapidly developing energy storage technology that provides significant robustness and efficiency benefits over alternative energy storage technologies [2]. In particular, compared with electrochemical batteries, supercapacitors can typically survive 100 to 1000 times as many charge-discharge cycles before there is significant degradation of capacity or efficiency [3]. This is a substantial benefit for remote systems because it can reduce the frequency of maintenance visits to remotely deployed nodes and thereby achieve greater cost-effectiveness. Thus far, supercapacitors have been commonly applied for buffering energy over short durations, e. g. supercapacitorbattery hybrid storage $[4,5]$, and regenerative breaking $[6,7,8]$. For these applications, peak power and short-term efficiency are supercapacitors' most important strengths.

The proposed work targets long-term energy storage applications where accurate energy awareness is important. For example, a remotely deployed system can rely on solar energy harvesting. Without a reliable connection to the electrical grid, accurate knowledge of a supercapacitor's SOC (state-of-charge) is critical to know how much energy remains available to the system $[9,10]$. Longterm energy storage applications are also motivated by the robustness and durability benefits of supercapacitors. These benefits have been used to prototype a supercapacitor-based, WSN (wireless sensor node) with 20 years of projected service-free lifetime [11].

Tracking SOC can also be important when supercapacitors are used in banks of multiple devices in series or parallel configurations. These configurations benefit from load balancing in order to prevent specific cells from over-charging and suffering premature capacity degradation [12]. However, if SOC is not 
accounted for it can be difficult to perform on-line measurements of capacity degradation for the individual cells, or make real-time load balancing decisions.

Kalman tracking for a supercapacitor's SOC is proposed to exploit the energy awareness benefits of supercapacitors. Compared to rechargeable batteries, the stored energy in a supercapacitor is more directly related to its terminal voltage, $v_{\mathrm{sc}}$, via the capacitance, $C$. However, the simple model, $1 / 2 C v_{\mathrm{sc}}^{2}$, for stored energy neglects important non-ideal behavior: both charge redistribution and leakage affect storage capacity and the net portion of the energy that is available to a system after internal losses. It is shown that the total energy needed to fully charge a supercapacitor to its maximum voltage can vary up to $23 \%$ depending on the power level at which a supercapacitor is charged. This variation in capacity cannot be accounted for by the simple model. Extensive work has been done to model supercapacitor behavior $[13,14,15,16,17,18,19,20,21]$, but this paper goes to the next step: translating these models into usable energy awareness. The proposed Kalman tracking technique uses the three branch equivalent circuit [15] to account for this non-ideal capacity variability. The Kalman filter balances uncertainty by weighting the correction due to each new observation on the estimate of the circuit's SOC.

A common difficulty of Kalman tracking is the accuracy of the model. The parameter estimation technique proposed here provides a novel method to fit the parameters of the three branch equivalent circuit. The proposed technique does not require specific tests to be performed in the field or before the device is deployed: fitting is able to utilize the actual current profiles encountered by the application, provided significant power variability occurs. Prior methods for parameter estimation have relied on electrochemical impedance spectroscopy (EIS), which requires specialized test equipment and cannot be performed in the field $[22,23,24]$, or have been designed to only fit the capacitance and series resistance, but not the parameters for redistribution [25].

In addition to describing the proposed parameter estimation technique and Kalman tracking methodology, this paper also presents experimental validation of both techniques for $50 \mathrm{~F}$ supercapacitors and parameter estimation for $5 \mathrm{~F}$, 
$10 \mathrm{~F}$ and $350 \mathrm{~F}$ supercapacitors.

This paper begins with background and modeling for supercapacitors in Section 2. Sections 3 and 4 then present the proposed parameter estimation and Kalman tracking techniques. Section 5 evaluates the performance of each technique as compared to the simple capacitive model. Results and discussion of the experiments are given in Section 6. Conclusions and contributions are summarized in Section 7.

\section{Modeling}

Charge storage in supercapacitors relies primarily on two phenomena: the EDL (electric double layer) and pseudocapacitance [13], that are illustrated in Fig. 1a. Voltage applied across the supercapacitor terminals pushes charge to the surface of the electrode material forming a surface layer. Oppositely charged ions in the electrolyte are, in turn, attracted by the surface charge forming a second layer. The two layers make up the EDL capacitance. Reversible chemical reactions store additional charge on the surface of the electrodes and contribute pseudocapacitance. Much higher capacitances than conventional electrolytic devices are obtained because of the small distance (comparable to atomic radii) between opposite charges and the large surface area of the porous electrodes.

A simple capacitive model fails to account for three sources of non ideal behavior in supercapacitors: both the EDL and pseudocapacitance are voltage dependent; the diffusion of ions into the porous materials is not instantaneous; and there is spontaneous leakage of stored charge. Diffusion, or charge redistribution, is observable after a charging current into a supercapacitor is discon-

tinued. Diffusion of ions in the electrolyte causes the terminal voltage, $v_{\mathrm{sc}}$, to spontaneously decay. Diffusion push ions away from regions of high concentration near the readily accessible (and more quickly charged) portions of the porous surface. Modeling a supercapcitor's SOC accounts for charge redistribution as the penetration of stored charge into a string of resistive-capacitive (RC) transmission line storage elements [14] as in Fig. 1a, or simplified as an array of 
time constants in parallel RC branches [15] as in Fig. 1b. Leakage has been simply modeled as a fixed parallel resistance [15], or a variable resistance depending on factors such as the supercapacitor's voltage, temperature and internal state $[16,26,17,18,27]$.

Prior work has shown that a simple capacitive model can provide adequate energy awareness for low powered solar-supercapacitor WSN (wireless sensor nodes) $[28,29]$. Online parameter estimation has also been demonstrated for a single branch model [30]. Although the simple model is preferable because of the limited computational capabilities of these systems, results in [29] show significant improvement when the fixed capacitance, $C_{\text {simple }}$, is tuned to the specific discharge speed, similar to the manner in which three branch equivalent circuit intrinsically accounts for the effect of charging speed on SOC. Modeling a supercapacitor using the three branch equivalent circuit is especially important for higher powered systems with greater power variability than WSN.

The impact of both redistribution and leakage when the charging current, $i_{\mathrm{sc}}$, is varied over a wider range of power is shown in Fig. 2. The observed supercapacitor behavior is compared to the baseline, simple capacitive model for stored energy,

$$
E=\frac{1}{2} C_{\text {simple }} v_{\mathrm{sc}}^{2}
$$

where $v_{\mathrm{sc}}$ is the terminal voltage for the supercapacitor, and the capacitance, $C_{\text {simple }}$, is fitted to the average slope of the observed data,

$$
C_{\text {simple }}=\left(\frac{1}{N} \sum_{n=1}^{N} \frac{d v_{\mathrm{sc}}}{d q}\right)^{-1} .
$$

For each of the $N$ observations in (2), taken over the three durations of charging in Fig. 2, the differential charge, $d q$, is calculated as $i_{\mathrm{sc}} d t$, where $i_{\mathrm{sc}}$ denotes the (constant) charging current and $t$ denotes time. In terms of energy awareness, Fig. 2 shows that the total energy needed to charge the 50F supercapacitor,

$$
E_{\mathrm{obs}}=\int v_{\mathrm{sc}} i_{\mathrm{sc}} d t=\int v_{\mathrm{sc}} d q
$$

varies between $188 \mathrm{~J}$ and $231 \mathrm{~J}$ (23\% variation) even though the supercapacitor is charged to the same terminal voltage, and only the charging current, $i_{\mathrm{sc}}$, 
is varied. For a $350 \mathrm{~F}$ supercapacitor, the measured value of $E_{\text {obs }}$ varies from $1.18 \mathrm{~kJ}$ to $1.31 \mathrm{~kJ}$ ( $11 \%$ variation) despite the proportionally smaller range of charging currents tested for the larger supercapacitor. Redistribution predicts this variation in charge storage because the storage capacity of the supercapacitor's porous electrode surfaces is more fully accessed over the longer duration of charging for the lower currents. Redistribution in Fig. 2 shows that the decline in $v_{\mathrm{sc}}$ after charging stops is proportional to the speed of charging.

The proposed Kalman technique uses a simple fixed resistance to model leakage, and focuses on tracking the supercapacitor's SOC due to redistribution using the three branch equivalent circuit [15]. The real world implications of tracking a supercapacitor's SOC are motivated by the power variability shown in Fig. 3, for a system using supercapacitors to buffer solar energy harvesting. Other work $[31,32]$ has applied battery modeling to supercapacitors. However, supercapacitors do not require the complexity of these models devoted to modeling hysteresis and the nonlinear relationship between a battery's terminal voltage and SOC. For predicting short-term supercapacitor performance, it is beneficial to use more detailed models such as the transmission line elements in Fig. 1a that are accurate over much wider frequency ranges and tuned using electrochemical impedance spectroscopy [22, 23, 33]. For our target applications the three branch equivalent circuit provides a good balance between accuracy, complexity, and the ability to use on-line parameter estimation. Prior work has used the three branch equivalent circuit to model charge redistribution [34], but this method did not consider SOC tracking or on-line parameter estimation.

\section{Parameter Estimation Method}

Estimating the parameters of the three branch equivalent circuit is nontrivial due to the fact that the internal state is not observable. Linear LMSE (least mean square error) fitting is proposed for parameter identification because $i_{\mathrm{sc}}$ in the three branch equivalent circuit in Fig. 1b can be fitted as a linear combi- 
nation of the branch currents,

$$
i_{\mathrm{sc}}=C_{1} \frac{d v_{\mathrm{sc}}}{d t}+C_{\mathrm{var}} v_{\mathrm{sc}} \frac{d v_{\mathrm{sc}}}{d t}+\frac{1}{R_{2}}\left(v_{\mathrm{sc}}-v_{2}\right)+\frac{1}{R_{3}}\left(v_{\mathrm{sc}}-v_{3}\right),
$$

where $C_{1}$ and $C_{\mathrm{var}} v_{\mathrm{sc}}$ are the fixed and voltage dependent capacitances included in the first branch and, $R_{2}, R_{3}$ and $v_{2}, v_{3}$ are the resistances and voltages in the second and third branches, each as labeled in Fig. 1b. Leakage is neglected as it is much less significant than redistribution in the short term.

The first and second terms in (4), accurately approximate the current in the first branch using the observable slope of $v_{\mathrm{sc}}$ rather than the true slope of hidden voltage, $v_{1}$. This is a safe assumption because the voltage drop between $v_{\mathrm{sc}}$ and $v_{1}$ due to $R_{\text {serial }}$ is small. Jumps in $v_{\mathrm{sc}}$ when $i_{\mathrm{sc}}$ switches on or off are omitted in order to avoid the discontinuities in $d v_{\mathrm{sc}} / d t$. The last two terms in (4), for the current from the second and third branches, depend on the low pass filtered branch voltages, $v_{2}$ and $v_{3}$, with time constants, $\tau_{2}$ and $\tau_{3}$,

$$
\begin{aligned}
& v_{2}(t)=\frac{1}{\tau_{2}} \int_{0}^{t} v_{\mathrm{sc}}\left(t^{\prime}\right) e^{\frac{-\left(t-t^{\prime}\right)}{\tau_{2}}} d t^{\prime}, \\
& v_{3}(t)=\frac{1}{\tau_{3}} \int_{0}^{t} v_{\mathrm{sc}}\left(t^{\prime}\right) e^{\frac{-\left(t-t^{\prime}\right)}{\tau_{3}}} d t^{\prime} .
\end{aligned}
$$

Simplifying (4) into matrix form, $i_{\mathrm{sc}}=\mathbf{U} \boldsymbol{\beta}$, the circuit parameters, $\boldsymbol{\beta}=$ $\left[C_{1}, C_{\mathrm{var}}, 1 / R_{2}, 1 / R_{3}\right]^{\mathrm{T}}$, can be estimated as,

$$
\hat{\boldsymbol{\beta}}=\left(\mathbf{U}^{\mathrm{T}} \mathbf{U}\right)^{-1} \mathbf{U}^{\mathrm{T}} i_{\mathrm{sc}} .
$$

The predictor matrix, $\mathbf{U}$, depends only on $v_{\mathrm{sc}}$, which is observable, and $\tau_{2}$ and $\tau_{3}$ which are determined separately,

$$
\mathbf{U}=\left[\frac{d v_{\mathrm{sc}}}{d t}, v_{\mathrm{sc}} \frac{d v_{\mathrm{sc}}}{d t}, v_{\mathrm{sc}}-v_{2}, v_{\mathrm{sc}}-v_{3}\right] .
$$

After using LMSE to calculate $\hat{\boldsymbol{\beta}}$, the remaining circuit parameters are: $R_{\text {serial }}, C_{2}, C_{3}$, and $R_{\text {leak }}$. $R_{\text {serial }}$ is found by observing the change, $\Delta V$ in $v_{\mathrm{sc}}$, that occurs in response to the change, $\Delta I$ when $i_{\mathrm{sc}}$ is switched off,

$$
R_{\text {serial }}=\frac{\Delta V}{\Delta I} .
$$


The capacitances, $C_{2}$ and $C_{3}$, are calculated from the time constants,

$$
C_{2}=\frac{1}{R_{2}} \tau_{2}, \quad \text { and } \quad C_{3}=\frac{1}{R_{3}} \tau_{3} .
$$

The time constants, $\tau_{2}$ and $\tau_{3}$, are not estimated by the LMSE procedure, but are needed to calculate, $v_{2}$ and $v_{3}$ using (5) and (6). As in prior work [15], it is possible to make assumptions that dictate the timescale of each branch. This is comparable to fixing $\tau_{2}$ and $\tau_{3}$ heuristically. Alternatively, the LMSE procedure is performed iteratively to search over different values for $\tau_{2}$ and $\tau_{3}$ that minimize the mean square error in LMSE. For the iterative search, it is important that the test data for $v_{\mathrm{sc}}$ and $i_{\mathrm{sc}}$ contain a rich variation of supercapacitor behavior over different time scales. The last parameter, $R_{\text {leak }}$, models long term leakage, and cannot be observed over a short duration. A fixed value for $R_{\text {leak }}$ can be fitted to the observed decline in $v_{\mathrm{sc}}$ after redistribution is given enough time to ensure that the supercapacitor is in an equilibrium state. However, the most significant decline in $v_{\mathrm{sc}}$ that occurs immediately after charging stops is be well modeled by charge redistribution. Because the impact of leakage on energy awareness is overshadowed by redistribution for harvesting applications, $R_{\text {leak }}$ is preset generically based on the manufacturer's specifications.

While calculating $\hat{\boldsymbol{\beta}}$, it is useful to realize that LMSE penalizes the squared value of the residual error in the fitted current. When the scale of the current into supercapacitor varies by orders of magnitude, it is useful to normalize both $i_{\mathrm{sc}}$ and $\mathbf{U}$ by $i_{\mathrm{sc}}^{-1}$ so errors at high current do not overshadow all else. LMSE also weights each sample evenly, so downsampling in proportion to $i_{\mathrm{sc}}^{-1}$ was found to improve results. This technique devotes equal numbers of samples to each

charging test regardless of the actual duration needed for each test current to charge the supercapacitor.

\section{Kalman Tracking Method}

Using the three branch equivalent circuit, the supercapacitor's SOC at each time step, $t_{n}$, can be represented as a vector of the voltages across each capaci- 
tance of the equivalent circuit shown in Fig. 1b,

$$
\mathbf{x}_{n}=\left[v_{1}\left(t_{n}\right), \quad v_{2}\left(t_{n}\right), \quad v_{3}\left(t_{n}\right)\right]^{\mathrm{T}} .
$$

Tracking $\mathbf{x}_{n}$, via the classic Kalman technique [36, Chap. 13], relies on the fact that random Gaussian inputs to a linear dynamic system will produce a random Gaussian output. Consequently, the three branch equivalent circuit is modeled as a linear system,

$$
\begin{gathered}
\mathbf{x}_{n}=\mathbf{F} \mathbf{x}_{n-1}+\mathbf{B} \mathbf{u}_{n}+\mathbf{w}_{n} \\
\mathbf{z}_{n}=\mathbf{H} \mathbf{x}_{n}+\mathbf{D} \mathbf{u}_{n}+\mathbf{v}_{n}
\end{gathered}
$$

Using $\mathcal{N}(\mu, \boldsymbol{\Sigma})$ to represent a multivariate Gaussian distribution with mean, $\mu$, and covariance matrix, $\boldsymbol{\Sigma}$, uncertainty is modeled by the additive white Gaussian noise (AWGN) signals,

$$
\begin{aligned}
& \mathbf{w}_{n} \sim \mathcal{N}(\mathbf{0}, \mathbf{Q}) \\
& \mathbf{v}_{n} \sim \mathcal{N}(\mathbf{0}, \mathbf{R})
\end{aligned}
$$

The assumption of zero means for the noise processes in the formulation is not particularly limiting and the formulation can be extended to address the nonzero mean situations as is standardly done in statistical signal processing.

For the three branch equivalent circuit, the input, $\mathbf{u}_{n}$, is the average value of $i_{\mathrm{sc}}$ into the supercapacitor over the time $t_{n-1}$ to $t_{n}$. The output, $\mathbf{z}_{n}$, is $v_{\mathrm{sc}}$ measured at time $t_{n}$. The matrices, $\mathbf{F}, \mathbf{B}, \mathbf{H}$ and $\mathbf{D}$, are found by solving the system of linear ODE (ordinary differential equations) from the three branch equivalent circuit,

$$
\begin{aligned}
\frac{d}{d t} \mathbf{x}(t) & =\mathbf{F}_{\Delta} \mathbf{x}(t)+\mathbf{B}_{\Delta} \mathbf{u}(t) \\
\mathbf{z}(t) & =\mathbf{H} \mathbf{x}(t)+\mathbf{D} \mathbf{u}(t)
\end{aligned}
$$

The matrices, $\mathbf{F}_{\Delta}, \mathbf{B}_{\Delta}, \mathbf{H}$, and $\mathbf{D}$, are given in Table 1. The solution, $\mathbf{x}_{n}$, for the system of ODE is a sum of the homogeneous, $\mathbf{x}_{\text {homo }}$ and particular solutions, 
$\mathbf{x}_{\text {part }}$,

$$
\begin{aligned}
\mathbf{x}_{\text {homo }}\left(t_{n}\right) & =e^{\left(t_{n}-t_{n-1}\right) \mathbf{F}_{\Delta}} \cdot \mathbf{x}\left(t_{n-1}\right), \\
\mathbf{x}_{\mathrm{part}}\left(t_{n}\right) & =\int_{t_{n-1}}^{t_{n}} e^{\left(t^{\prime}-t_{n-1}\right) \mathbf{F}_{\Delta}} \cdot \mathbf{B}_{\Delta} \mathbf{u}\left(t^{\prime}\right) d t^{\prime},
\end{aligned}
$$

where both (18) and (19) rely on the standard definition of the matrix exponential, $e^{\mathbf{X}}=\sum_{k=0}^{\infty} 1 / k ! \mathbf{X}^{k}$. Using (18) and (19) respectively, the transition matrix, $\mathbf{F}$, and input matrix, $\mathbf{B}$, are,

$$
\begin{gathered}
\mathbf{F}=e^{\left(t_{n}-t_{n-1}\right) \mathbf{F}_{\Delta}}, \\
\mathbf{B}=\mathbf{F}_{\Delta}^{-1}\left(e^{\left(t_{n}-t_{n-1}\right) \mathbf{F}_{\Delta}}-\mathbf{I}\right) \cdot \mathbf{B}_{\Delta} .
\end{gathered}
$$

The integral appearing in (19) has been simplified using properties of matrix exponentiation. The output matrices, $\mathbf{H}$ and $\mathbf{D}$, are taken directly from the equivalent circuit as listed in Table 1.

Due to the voltage dependent capacitance, $\tau_{1}$ in $\mathbf{F}_{\Delta}$ is not fixed and (16) is not strictly a linear system. However, the variation of $\tau_{1}$ over the time step from $t_{n-1}$ to $t_{n}$ is minimal. The approximate linear solution, given by (18) and (19), and referred to as the EKF (extended Kalman filter), provides accurate results. For many other applications that require models with greater non-linearity, the alternative SPKF (sigma point Kalman filter) is needed [37].

The covariance matrices $\mathbf{Q}$ and $\mathbf{R}$ are set heuristically to balance convergence speed against noise tolerance. Specifically, we set $\mathbf{Q}$ and $\mathbf{R}$ as diagonal matrices,

$$
\begin{gathered}
\mathbf{Q}=\alpha\left(\left|i_{\mathrm{sc}}\right|+\epsilon\right)\left(t_{n}-t_{n-1}\right)\left[\begin{array}{ccc}
\frac{R_{\|}}{\tau_{1}} & 0 & 0 \\
0 & \frac{R_{\|}}{\tau_{2}} & 0 \\
0 & 0 & \frac{R_{\|}}{\tau_{3}}
\end{array}\right], \\
\mathbf{R}=\alpha\left(\left|i_{\mathrm{sc}}\right|+\epsilon\right)\left[R_{\|}\right],
\end{gathered}
$$

where $\epsilon$ is a small value introduced to ensure invertibility and $\alpha$ is the gain factor. Intuitively, (22) assumes an uncertainty in each predicted state variable that is roughly proportional to the magnitude of its change between adjacent 
observations; and (23) assumes an uncertainty in the predicted output that is roughly proportional to the magnitude of the resistive voltage drop due to the input current. Values of $\epsilon=0.01$ and $\alpha=0.01$ were used and the method was not found to be particularly sensitive to variations in these parameters.

For each time step, $t_{n}$, Kalman filtering calculates the MAP (maximum a posteriori) estimate of the state, $\hat{\mathbf{x}}_{n}$, and its error covariance matrix, $\hat{\mathbf{P}}_{n}$,

$$
\begin{gathered}
\hat{\mathbf{x}}_{n}=\tilde{\mathbf{x}}_{n}+\mathbf{K}\left(\mathbf{z}_{n}-\mathbf{H} \tilde{\mathbf{x}}_{n}-\mathbf{D} \mathbf{u}_{n}\right) \\
\hat{\mathbf{P}}_{n}=\tilde{\mathbf{P}}_{n}-\mathbf{K H} \tilde{\mathbf{P}}_{n}
\end{gathered}
$$

where the Kalman gain, $\mathbf{K}$, is,

$$
\mathbf{K}=\tilde{\mathbf{P}}_{n} \mathbf{H}^{\mathrm{T}}\left(\mathbf{H} \tilde{\mathbf{P}}_{n} \mathbf{H}^{\mathrm{T}}+\mathbf{R}\right)^{-1}
$$

and the mean, $\tilde{\mathbf{x}}_{n}$ and covariance, $\tilde{\mathbf{P}}_{n}$, of the predicted prior distribution for $\mathbf{x}_{n}$ are calculated as,

$$
\begin{aligned}
\tilde{\mathbf{x}}_{n} & =\mathbf{F} \hat{\mathbf{x}}_{n-1}+\mathbf{B} \mathbf{u}_{n}, \\
\tilde{\mathbf{P}}_{n} & =\mathbf{F} \hat{\mathbf{P}}_{n-1} \mathbf{F}^{\mathrm{T}}+\mathbf{Q} .
\end{aligned}
$$

A quick discussion of these Kalman equations is given in Section S1 in the supplementary material.

\section{Evaluation Method}

Evaluation demonstrates how both of the proposed methods, parameter estimation and Kalman tracking, each improve energy awareness. Performance is characterized by the error between the models' predictions and the actual observed energy that would be available to an application.

As a baseline, the stored energy in a supercapacitor is predicted from $v_{\mathrm{sc}}$ using the simple capacitive model,

$$
E=\frac{1}{2} C_{\text {simple }} v_{\mathrm{sc}}^{2}
$$


Alternatively, $E$ is predicted from a supercapacitor's SOC using the three branch equivalent circuit model,

$$
E=\frac{1}{2} C_{1} v_{1}^{2}+\frac{1}{3} C_{\mathrm{var}} v_{1}^{3}+\frac{1}{2} C_{2} v_{2}^{2}+\frac{1}{2} C_{3} v_{3}^{2} .
$$

Intuitively, (29) and (30) can be validated by measuring the total energy that is recovered by completely discharging a supercapacitor. However, long term charge storage in a supercapacitor may still hold significant energy even after $v_{\mathrm{sc}}$ is discharged to zero volts. This unaccounted-for energy complicates validating $E$ directly.

Rather than completely discharging a supercapacitor, energy awareness is validated by predicting the change in a supercapacitor's stored energy, $\Delta E$, while a supercapacitor is charged and discharged to various given final voltages. This corresponds to predicting how much energy would be available to an application before $v_{\mathrm{sc}}$ falls below a minimum operating voltage. Additionally the three branch equivalent circuit predicts internal losses, $W$, by integrating the power dissipated from each of the model's resistors. The simple model of (29) does not include internal losses. The observed energy, $E_{\text {obs }}$, that can be accurately measured is predicted by the model as,

$$
E_{\mathrm{obs}}=\Delta E+W
$$

Both the proposed methods and the simple capacitive model are tested by estimating $\Delta E$ over durations of constant current charging $(\Delta E>0)$ and discharging $(\Delta E<0)$, and durations of redistribution where $i_{\mathrm{sc}}$ is zero. First, the proposed Kalman tracking method estimates the initial SOC. Starting at this initial SOC, equations (12) and (13) are then used to predict the supercapacitor's behavior until $v_{\mathrm{sc}}$ reaches the same final voltage as the observed data. Finally, equations (29) and (30) are used to predict $E$ before and after the duration of constant current. The change in stored energy, $\Delta E$, is compared against the observed energy,

$$
\text { error }=E_{\mathrm{obs}}-(\Delta E+W) .
$$


In order to validate parameter estimation, the supercapacitor is charged from a known completely discharged state, such that there is no need to use tracking to estimate the initial state. Before each test the supercapacitor is discharged and then left overnight with its terminals short circuited. Because the initial $E$ is zero, $E_{\text {obs }}$ can be directly compared to the stored energy, $E$.

\section{Results and Discussion}

The experimental setup for charging and discharging the supercapacitors is shown in Fig. 4. Testing is conducted for 5F, 50F, and 350F Maxwell BoostCap supercapacitors [38], and a 10F device from Illinois Capacitor [39]. The range of charging currents for the $50 \mathrm{~F}$ supercapacitor is set to coincide with the variability range of solar energy harvesting shown in Fig. 3. Charging currents are supplied by a Nemic-Lambda ZUP (36V-24A) current source. The supercapacitor's terminal voltage is measured using an ADC (analog to digital converter) on a Microchip PIC16F1783 PIC (programmable interface controller). The PIC also automatically controls the timing. A power MOSFET allows the PIC to disconnect the charging current when the supercapacitor reaches its rated voltage. Discharging the supercapacitor is done by a TekPower 3710A DC Electronic Load $(0-360 \mathrm{~V} / 150 \mathrm{~W})$ in constant current mode. The PIC controls a second MOSFET that disconnects the load when $v_{\text {sc }}$ falls below $.35 \mathrm{~V}$ because the constant load current becomes unreliable below that point.

\subsection{Parameter Estimation}

Because the true parameters of a physical supercapacitor are not accessible, parameter estimation is first tested using simulated data rather than observed data. Using known parameters from prior work [15], equations (12) and (13) simulate the SOC and $v_{\mathrm{sc}}$ of a $470 \mathrm{~F}$ DLC supercapacitor as it is charged from a completely discharged state to its rated voltage (2.3V). Three different constant currents are used to charge the supercapacitor at approximately $5 \%, 0.5 \%$, and $0.05 \%$ of its listed short circuit current. Using the simulated $i_{\mathrm{sc}}$ and $v_{\mathrm{sc}}$ data, 
equations (7) and (8) are able to accurately recover the true parameters used to generate the simulated data. Table 2 shows the accuracy of the estimated parameters. The proposed LMSE method is able to improve on the accuracy of the prior parameter estimation method [15] because it does not rely on the assumption that significant current only flows into one branch of the circuit at a time. This allows the proposed method to more accurately distinguish the simultaneous effects of charge redistribution and voltage dependent capacitance. Consequently, the proposed parameter estimation improves the accuracy of both $C_{\mathrm{var}}$ and the parameters of the second and third branches. The average deviation between the fitted parameters and the simulation groundtruth is decreased from $13 \%$ error for the method of [15] to less than $2 \%$ error for the proposed method.

In terms of energy awareness for physical supercapacitors, the benefits of the three three branch equivalent circuit model are shown in Fig. 5. Parameters for the $5 \mathrm{~F}, 10 \mathrm{~F}, 50 \mathrm{~F}$, and $350 \mathrm{~F}$ supercapacitors are estimated using the procedure given in Section 3. The required input data, $i_{\mathrm{sc}}$ and $v_{\mathrm{sc}}$, is a concatenation of $10 \mathrm{~mA}, 100 \mathrm{~mA}$, and $1 \mathrm{~A}$ charging patterns for the $10 \mathrm{~F}$ supercapacitor; $100 \mathrm{~mA}$, $1 \mathrm{~A}$, and $10 \mathrm{~A}$ charging patterns shown in Fig. 6 for the 50F supercapacitor; and $500 \mathrm{~mA}, 2 \mathrm{~A}, 10 \mathrm{~A}$, and $20 \mathrm{~A}$ charging patterns for the $350 \mathrm{~F}$ supercapacitor. Additionally, a $70 \mathrm{~A}$ charging behavior is modeled for the $350 \mathrm{~F}$, but observed data was not collected for that current. The fitted parameters are given in Table 3 , and used to estimate $\Delta E$ and $W$ in Fig. 5 . In order to test the reliability of the proposed parameter estimation, multiple 50F supercapacitors are tested. As shown in Table S1 in the supplementary material, the fitted parameters across multiple $50 \mathrm{~F}$ devices are consistent within a reasonable tolerance that is expected for physical devices.

As compared to using the rated capacitance, $C_{\text {rated }}$, to predict the stored energy, the three branch equivalent circuit is able to account for the varying behavior of the supercapacitor at different charging currents. Even when the simple capacitance, $C_{\text {simple }}$, is fitted using the same charging data, a fixed capacitance underestimates $E$ at low currents when a greater portion of the charge 
storage on the supercapacitor's porous electrode surfaces is utilized, and overestimates $E$ at high current when a smaller portion is utilized. The simple capacitive models are also unable to model the energy, $W$, that is dissipated internally as waste. Neglecting $W$ causes the simple capacitive model to consistently underestimate $E$ even when $C_{\text {simple }}$ is fitted to $d v_{\text {sc }} / d t$. Cross-validation of the three branch equivalent circuit's ability to model $E$ for data not used for fitting is shown next for the SOC tracking technique.

\subsection{Kalman Tracking}

The energy awareness benefits of proposed Kalman methods are validated by tracking a supercapacitor's SOC as it is repeatedly charged and discharged. Because the supercapacitor is no longer in a known completely discharged state at the start of each charge cycle, the proposed Kalman tracking technique is used to track the SOC, as shown in Fig. 7. The exception is the very first 5A charging current where the supercapacitor starts from rest.

Using the estimated SOC at the start of each duration of charging, discharging and redistribution, the three branch equivalent circuit predicts $\Delta E$ that is transfered to or from the supercapacitor. Figure 8 compares the estimates of $\Delta E$ and $W$ against the observed energy $E_{\text {obs }}$. Using the three branch equivalent circuit model outperforms the simple models that use either the fixed capacitance, $C_{\text {rated }}$, from the datasheet, or $C_{\text {simple }}$, fitted to the observed data. The fixed capacitive models for energy awareness especially struggle during redistribution when $i_{\mathrm{sc}}$ is zero. These models cannot account for the decrease in $v_{\mathrm{sc}}$ unaccompanied by energy output during redistribution. At each point where the supercapacitor is maximally charged, the estimated energy available to an application would be subject to both the error during redistribution and discharging, for a total overestimation of up to $18 \mathrm{~J}$ by the simple model. The maximum total error for the Kalman tracking is 5.8J. As shown in the results for parameter estimation, the spontaneous decrease in $v_{\mathrm{sc}}$ is best modeled by the three branch equivalent circuit as opposed to more complex leakage models. Leakage models would have greater difficulty accounting for the systematic error 
of the simple capacitive model during the charging cycles that is well explained by the three branch equivalent circuit.

\section{Conclusions}

This paper has provided a novel technique to monitor the remaining energy stored in a supercapacitor by tracking its SOC using the three branch equivalent circuit model in conjunction with a Kalman tracker. Tracking a supercapacitor's SOC is shown to reduce the error in estimation of the net change in the buffered energy, $\Delta E_{\text {tot }}$ by $67 \%$ and $49 \%$ as compared to the simple capacitive model using the datasheet and fitting respectively to set $C_{\text {simple. It is shown }}$ that charge redistribution is the main source of the simple model's inaccuracy, justifying the use of the three branch equivalent circuit to model the supercapacitor's SOC. With an eye towards applicability, a novel parameter estimation technique is also proposed such that the parameters of the three branch circuit can be calculated from arbitrary current and voltage profiles observed while the supercapacitor operates normally in its application setting. The two significant benefits of this on-line parameter estimation are: 1) there is no need to take device off-line to measure the parameters or possible degradation of capacity over time, or perform specific tests before individual systems are deployed to account for variation in capacity due to manufacturing tolerances; 2) on-line parameter estimation allows the model to be fitted to the same data it is tracking such that any biased measurements due to hardware on a specific system will be incorporated into the parameters. The performance of both proposed techniques, parameter estimation and SOC tracking, are physically validated for $5 \mathrm{~F}, 10 \mathrm{~F}, 50 \mathrm{~F}$ and $350 \mathrm{~F}$ supercapacitors and shown to outperform the simple capacitive model for supercapacitor behavior.

\section{References}

[1] A. Nadeau, G. Sharma, T. Soyata, State-of-charge estimation for supercapacitors: a Kalman filtering formulation, in: Proc. IEEE Intl. Conf. Acous- 
tics Speech and Sig. Proc., (ICASSP), Florence, Italy, 2014, pp. 2194-2198. doi:10.1109/ICASSP.2014.6853988.

[2] P. Simon, Y. Gogotsi, B. Dunn, Where do batteries end and supercapacitors begin?, Science Magazine 343 (2014) 1210-1211. doi:10.1126/science.1249625.

[3] A. Gee, F. Robinson, R. Dunn, Analysis of battery lifetime extension in a small-scale wind-energy system using supercapacitors, IEEE Trans. Energy Convers. 28 (1) (2013) 24-33. doi:10.1109/TEC.2012.2228195.

[4] S. Lukic, S. Wirasingha, F. Rodriguez, J. Cao, A. Emadi, Power management of an ultracapacitor/battery hybrid energy storage system in an HEV, in: IEEE Vehicle Power and Propulsion Conference (VPPC), 2006, pp. 1-6. doi:10.1109/VPPC.2006.364357.

[5] H. Jia, Y. Mu, Y. Qi, A statistical model to determine the capacity of batterysupercapacitor hybrid energy storage system in autonomous microgrid, Int. J. of Electr. Pow. \& Energ. Sys. 54 (0) (2014) 516-524. doi:http://dx.doi.org/10.1016/j.ijepes.2013.07.025.

[6] J. Dixon, M. Ortuzar, Ultracapacitors + DC-DC converters in regenerative braking system, IEEE Aerospace and Electronic Sys. Mag. 17 (8) (2002) 16-21. doi:10.1109/MAES.2002.1028079.

[7] J. P. Torreglosa, P. Garcia, L. M. Fernandez, F. Jurado, Predictive control for the energy management of a fuel-cell-batterysupercapacitor tramway, IEEE Trans. Ind. Informat. 10 (1) (2014) 276-285. doi:10.1109/TII.2013.2245140.

[8] Q. Li, W. Chen, Z. Liu, M. Li, L. Ma, Development of energy management system based on a power sharing strategy for a fuel cell-batterysupercapacitor hybrid tramway, J. Power Sources 279 (0) (2015) 267-280, 9th International Conference on Lead-Acid Batteries \{LABAT\} 2014. doi:10.1016/j.jpowsour.2014.12.042. 
[9] A. Fahad, T. Soyata, T. Wang, G. Sharma, W. Heinzelman, K. Shen, SOLARCAP: super capacitor buffering of solar energy for self-sustainable field systems, in: Proceedings of the 25th IEEE International System-onChip Conference (IEEE SOCC), Niagara Falls, NY, 2012, pp. 236-241. doi:10.1109/SOCC.2012.6398354.

[10] M. Hassanalieragh, T. Soyata, A. Nadeau, G. Sharma, Solar-supercapacitor harvesting system design for energy-aware applications, in: Proceedings of the 27th IEEE International System-on-Chip Conference (IEEE SOCC), Las Vegas, NV, 2014, pp. 280-285. doi:10.1109/SOCC.2014.6948941.

[11] F. I. Simjee, P. H. Chou, Efficient charging of supercapacitors for extended lifetime of wireless sensor nodes, IEEE Trans. Power Electron. 23 (3) (2008) 1526-1536. doi:10.1109/TPEL.2008.921078.

[12] D. Linzen, S. Buller, E. Karden, R. W. De Doncker, Analysis and evaluation of charge-balancing circuits on performance, reliability, and lifetime of supercapacitor systems, IEEE Trans. Ind. Appl. 41 (5) (2005) 1135-1141. doi:10.1109/TIA.2005.853375.

[13] B. Conway, V. Birss, J. Wojtowicz, The role and utilization of pseudocapacitance for energy storage by supercapacitors, J. Power Sources 66 (1-2) (1997) 1-14. doi:10.1016/S0378-7753(96)02474-3.

[14] N. Bertrand, J. Sabatier, O. Briat, J.-M. Vinassa, Embedded fractional nonlinear supercapacitor model and its parametric estimation method, IEEE Trans. Ind. Appl. 57 (12) (2010) 3991-4000. doi:10.1109/TIE.2010.2076307.

[15] L. Zubieta, R. Bonert, Characterization of double-layer capacitors for power electronics applications, IEEE Trans. Ind. Appl. 36 (1) (2000) 199-205. doi:10.1109/28.821816.

[16] J. Niu, W. G. Pell, B. E. Conway, Requirements for performance characterization of c double-layer supercapacitors: Applications to a high 
specific-area c-cloth material, J. Power Sources 156 (2) (2006) 725-740. doi:10.1016/j.jpowsour.2005.06.002.

[17] Y. Zhang, H. Yang, Modeling and characterization of supercapacitors for wireless sensor network applications, J. Power Sources 196 (8) (2011) 41284135. doi:10.1016/j.jpowsour.2010.11.152.

[18] H. Yang, Y. Zhang, Self-discharge analysis and characterization of supercapacitors for environmentally powered wireless sensor network applications, J. Power Sources 196 (20) (2011) 8866-8873. doi:10.1016/j.jpowsour.2011.06.042.

[19] N. Devillers, S. Jemei, M.-C. Pra, D. Bienaim, F. Gustin, Review of characterization methods for supercapacitor modelling, J. Power Sources 246 (0) (2014) 596-608. doi:http://dx.doi.org/10.1016/j.jpowsour.2013.07.116.

[20] V. Sedlakova, J. Sikula, J. Majzner, P. Sedlak, T. Kuparowitz, B. Buergler, P. Vasina, Supercapacitor equivalent electrical circuit model based on charges redistribution by diffusion, J. Power Sources 286 (0) (2015) 58-65. doi:10.1016/j.jpowsour.2015.03.122.

[21] D. Torregrossa, M. Bahramipanah, E. Namor, R. Cherkaoui, M. Paolone, Improvement of dynamic modeling of supercapacitor by residual charge effect estimation, IEEE Trans. Ind. Appl. 61 (3) (2014) 1345-1354. doi:10.1109/TIE.2013.2259780.

[22] S. Buller, E. Karden, D. Kok, R. De Doncker, Modeling the dynamic behavior of supercapacitors using impedance spectroscopy, IEEE Trans. Ind. Appl. 38 (6) (2002) 1622-1626. doi:10.1109/TIA.2002.804762.

[23] S. Buller, M. Thele, R. De Doncker, E. Karden, Impedance-based simulation models of supercapacitors and Li-ion batteries for power electronic applications, IEEE Trans. Ind. Appl 41 (3) (2005) 742-747. doi:10.1109/TIA.2005.847280. 
[24] F. Rafik, H. Gualous, R. Gallay, A. Crausaz, A. Berthon, Frequency, thermal and voltage supercapacitor characterization and modeling, J. Power Sources 165 (2) (2007) 928-934. doi:10.1016/j.jpowsour.2006.12.021.

[25] A. Oukaour, M. Pouliquen, B. Tala-Ighil, H. Gualous, E. Pigeon, O. Gehan, B. Boudart, Supercapacitors aging diagnosis using least square algorithm, Microelectron. Reliab. 53 (9) (2013) 1638-1642. doi:10.1016/j.microrel.2013.07.032.

[26] Y. Diab, P. Venet, H. Gualous, G. Rojat, Self-discharge characterization and modeling of electrochemical capacitor used for power electronics applications, IEEE Trans. Power Electron. 24 (2) (2009) 510-517. doi:10.1109/TPEL.2008.2007116.

[27] H. Yang, Y. Zhang, Analysis of supercapacitor energy loss for power management in environmentally powered wireless sensor nodes, IEEE Trans. Power Electron. 28 (11) (2013) 5391-5403. doi:10.1109/TPEL.2013.2238683.

[28] C. Renner, V. Turau, CapLibrate: self-calibration of an energy harvesting power supply with supercapacitors, in: 23rd Intl. Conf. on Architecture of Computing Systems (ARCS), VDE, 2010, pp. 1-10.

[29] C. Renner, V. Turau, State-of-charge assessment for supercap-powered sensor nodes: Keep it simple stupid!, in: IEEE Ninth Intl. Conf. on Networked Sensing Systems (INSS), 2012, pp. 1-6. doi:10.1109/INSS.2012.6240582.

[30] A. Eddahech, M. Ayadi, O. Briat, J.-M. Vinassa, Online parameter identification for real-time supercapacitor performance estimation in automotive applications, Int. J. of Electr. Pow. \& Energ. Sys. 51 (0) (2013) 162-167. doi:10.1016/j.ijepes.2013.03.001.

[31] V. Johnson, Battery performance models in ADVISOR, J. Power Sources 110 (2) (2002) 321-329. 
[32] T. Markel, A. Brooker, T. Hendricks, V. Johnson, K. Kelly, B. Kramer, M. OKeefe, S. Sprik, K. Wipke, Advisor: a systems analysis tool for advanced vehicle modeling, J. Power Sources 110 (2) (2002) 255-266. doi:10.1016/S0378-7753(02)00189-1.

[33] S. Buller, E. Karden, D. Kok, R. De Doncker, Modeling the dynamic behavior of supercapacitors using impedance spectroscopy, in: Industry Applications Conference, Thirty-Sixth IAS Annual Meeting., Vol. 4, 2001, pp. 2500-2504. doi:10.1109/IAS.2001.955972.

[34] A. S. Weddell, G. V. Merrett, T. J. Kazmierski, B. M. Al-Hashimi, Accurate supercapacitor modeling for energy harvesting wireless sensor nodes, IEEE Trans. Circuits Syst. II, Exp. Briefs 58 (12) (2011) 911-915. doi:10.1109/TCSII.2011.2172712.

[35] R. Faranda, S. Leva, Energy comparison of MPPT techniques for PV systems, WSEAS Trans. on Power Systems 3 (6) (2008) 446-455.

[36] C. M. Bishop, Pattern recognition and machine learning, Information Science and Statistics, Springer, New York, NY, 2006.

[37] R. Van der Merwe, E. A. Wan, The square-root unscented Kalman filter for state and parameter-estimation, in: Proc. IEEE Intl. Conf. on Acoustics, Speech, and Sig, Proc. (ICASSP), Vol. 6, 2001, pp. 3461-3464. doi:10.1109/ICASSP.2001.940586.

[38] Maxwell Technologies, HC series ultracapacitors (2015).

URL www . maxwell.com/images/documents/hcseries_ds_1013793-9.pdf

[39] Illinois Capacitor Inc., DCN supercapacitors (2015).

URL

www.illinoiscapacitor.com/pdf/seriesDocuments/DCN series.pdf 


\section{List of Tables}

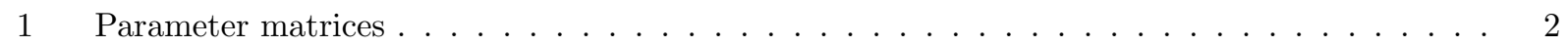

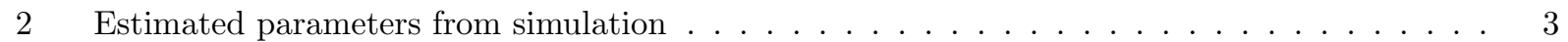

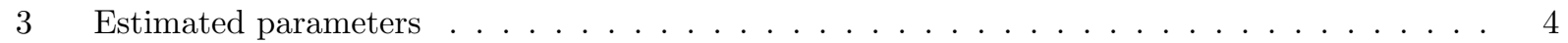

\section{List of Figures}

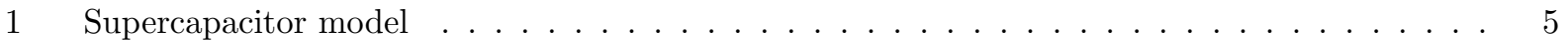

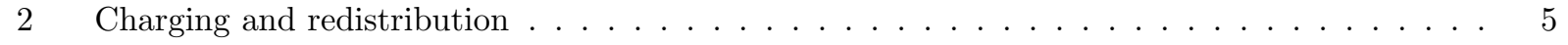

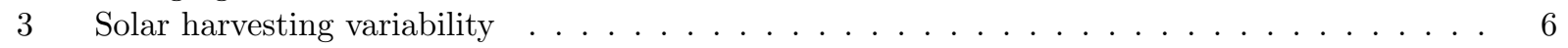

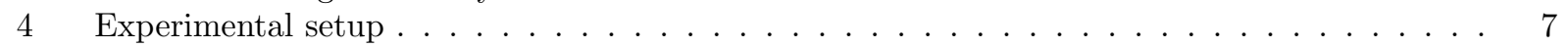

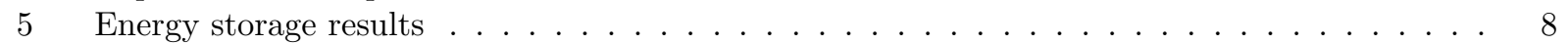

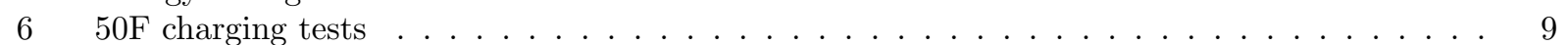

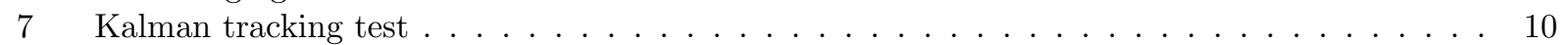

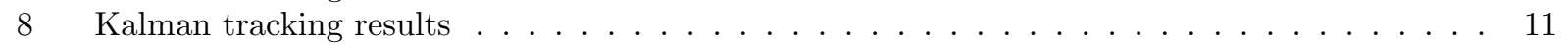




\begin{tabular}{c}
$\mathbf{F}_{\Delta}=\left[\begin{array}{ccc}\frac{1}{\tau_{1}} \frac{R_{\|}-R_{\text {serial }}}{R_{\text {serial }}} & \frac{1}{\tau_{1}} \frac{R_{\|}}{R_{2}} & \frac{1}{\tau_{1}} \frac{R_{\|}}{R_{3}} \\
\frac{1}{\tau_{2}} \frac{R_{\|}}{R_{\text {serial }}} & \frac{1}{\tau_{2}} \frac{R_{\|}-R_{2}}{R_{2}} & \frac{1}{\tau_{2}} \frac{R_{\|}}{R_{3}} \\
\frac{1}{\tau_{3}} \frac{R_{\|}}{R_{\text {serial }}} & \frac{1}{\tau_{3}} \frac{R_{\|}}{R_{2}} & \frac{1}{\tau_{3}} \frac{R_{\|}-R_{3}}{R_{3}}\end{array}\right], \quad \mathbf{B}_{\Delta}=\left[\begin{array}{c}\frac{1}{\tau_{1}} R_{\|} \\
\frac{1}{\tau_{2}} R_{\|} \\
\frac{1}{\tau_{3}} R_{\|}\end{array}\right]$, \\
$\mathbf{H}=\left[\begin{array}{lll}\frac{R_{\|}}{R_{\text {serial }}} & \frac{R_{\|}}{R_{2}} & \frac{R_{\|}}{R_{3}}\end{array}\right]$, \\
\hline$\tau_{1}=R_{\text {serial }}\left(C_{1}+C_{\text {var }} v_{1}\right), \quad \tau_{2}=R_{2} C_{2}$, \\
$\tau_{3}=R_{3} C_{3}, \quad R_{\|}=\left(R_{\text {serial }}^{-1}+R_{2}^{-1}+R_{3}^{-1}+R_{\text {leak }}^{-1}\right)^{-1}$. \\
\hline
\end{tabular}

Table 1: Parameter matrices for three branch equivalent circuit differential equation system in (16) and (17). 


\begin{tabular}{|l||c|cc|}
\hline \multicolumn{1}{|c||}{} & \multicolumn{3}{c|}{$470 \mathrm{~F}$ DLC (simulation) } \\
& truth & LMSE fit & ref. $[15]$ fit \\
\hline$C_{1}$ & $270 \mathrm{~F}$ & $273 \mathrm{~F}$ & $278 \mathrm{~F}$ \\
$C_{\text {var }}$ & $190 \frac{F}{V}$ & $191 \frac{F}{V}$ & $200 \frac{F}{V}$ \\
$R_{\text {serial }}$ & $2.5 \mathrm{~m} \Omega$ & $2.5 \mathrm{~m} \Omega$ & $2.5 \mathrm{~m} \Omega$ \\
\hline$C_{2}$ & $100 \mathrm{~F}$ & $98 \mathrm{~F}$ & $138 \mathrm{~F}$ \\
$R_{2}$ & $.90 \Omega$ & $.92 \Omega$ & $.87 \Omega$ \\
\hline$C_{3}$ & $220 \mathrm{~F}$ & $231 \mathrm{~F}$ & $143 \mathrm{~F}$ \\
$R_{3}$ & $5.2 \Omega$ & $5.2 \Omega$ & $5.5 \Omega$ \\
\hline$R_{\text {leak }}$ & $8 \mathrm{k} \Omega$ & \multicolumn{2}{c|}{ fitted separately $[15]$} \\
\hline
\end{tabular}

Table 2: The proposed (LMSE fit) technique is compared to prior work (ref. [15] fit) for estimating the parameters of the three branch equivalent circuit. The parameters are estimated using simulated measurement data for a $470 \mathrm{~F}$ DLC with (true) parameters specified in [15]. 


\begin{tabular}{|l||c|ccc|}
\hline & IL Cap [39] & \multicolumn{3}{|c|}{ Maxwell BCAP [38] } \\
$C_{\text {rated }}$ & $10 \mathrm{~F}$ & $5 \mathrm{~F}$ & $50 \mathrm{~F}$ & $350 \mathrm{~F}$ \\
\hline$C_{\text {simple }}$ & $13 \mathrm{~F}$ & $7.5 \mathrm{~F}$ & $57 \mathrm{~F}$ & $383 \mathrm{~F}$ \\
\hline$C_{1}$ & $8.8 \mathrm{~F}$ & $5.2 \mathrm{~F}$ & $40 \mathrm{~F}$ & $264 \mathrm{~F}$ \\
$C_{\text {var }}$ & $.63 \frac{F}{V}$ & $1.0 \frac{F}{V}$ & $9.1 \frac{F}{V}$ & $50 \frac{F}{V}$ \\
$R_{\text {serial }}$ & $39 \mathrm{~m} \Omega$ & $110 \mathrm{~m} \Omega$ & $22 \mathrm{~m} \Omega$ & $6.4 \mathrm{~m} \Omega$ \\
\hline$C_{2}$ & $.84 \mathrm{~F}$ & $.68 \mathrm{~F}$ & $2.2 \mathrm{~F}$ & $51 \mathrm{~F}$ \\
$R_{2}$ & $8.1 \Omega$ & $10 \Omega$ & $3.0 \Omega$ & $.13 \Omega$ \\
\hline$C_{3}$ & $6.0 \mathrm{~F}$ & $4.4 \mathrm{~F}$ & $11 \mathrm{~F}$ & $33 \mathrm{~F}$ \\
$R_{3}$ & $79 \Omega$ & $110 \Omega$ & $43 \Omega$ & $14 \Omega$ \\
\hline$R_{\text {leak }}$ & $90 \mathrm{k} \Omega$ & $180 \mathrm{k} \Omega$ & $36 \mathrm{k} \Omega$ & $9 \mathrm{k} \Omega$ \\
\hline
\end{tabular}

Table 3: The propose LMSE technique is used to estimate the parameters of the $5 \mathrm{~F}, 10 \mathrm{~F}, 50 \mathrm{~F}$, and $350 \mathrm{~F}$ supercapacitors using observed training data. 


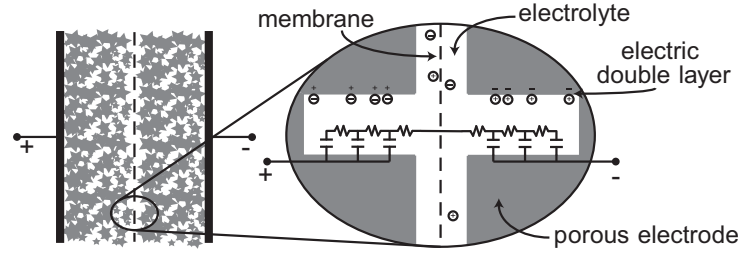

(a)

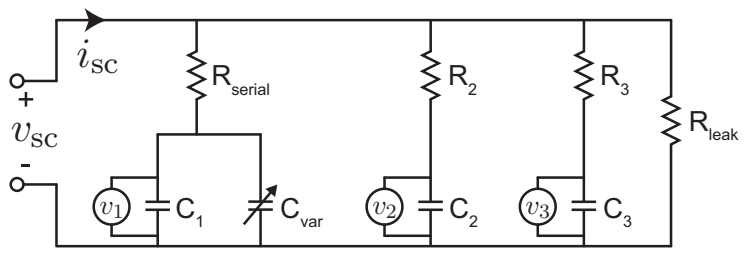

(b)

Figure 1: As shown in (a), each of a supercapacitor's terminals is connected to a porous electrode material such as activated carbon. The conductive electrodes are immersed in an electrolyte but separated by a membrane which only allows the ions in the electrolyte to pass through. Diffusion of the ions into the porous surface is modeled by the three branch equivalent circuit [15], shown in (b). Each branch has a time constant of increasing duration, $R_{\text {serial }} C_{1}<R_{2} C_{2}<R_{3} C_{3}$, modeling initial, intermediate, and long term behavior.
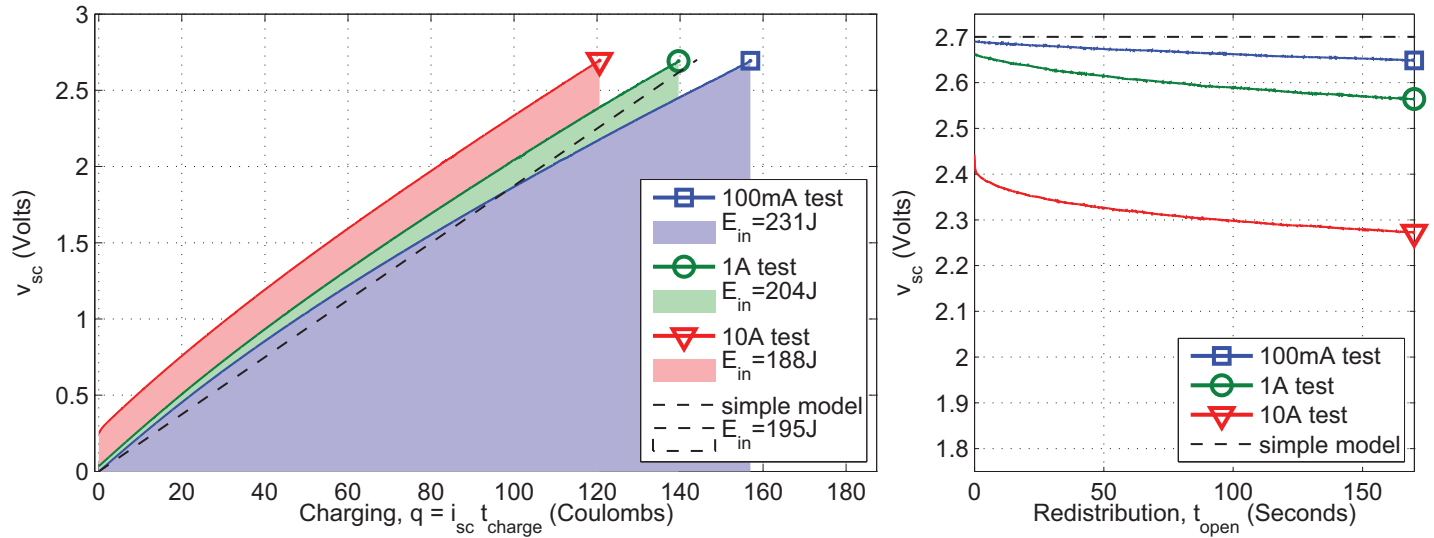

Figure 2: Charging, on the left, shows the terminal voltage of a 50F Maxwell Boostcap Ultracapacitor as it is charged with $100 \mathrm{~mA}, 1 \mathrm{~A}$, and $10 \mathrm{~A}$ constant currents. Because Charging is scaled to Coulombs, $q$, it is easy to see that a supercapacitor does not strictly follow the proportion, $v_{\mathrm{sc}}=1 / C \cdot q$, predicted by the simple model. The supercapacitor always starts in a completely discharged state, and current, $i_{\mathrm{sc}}$, is disconnected when $v_{\mathrm{sc}}$ reaches the device's rated voltage, $2.7 \mathrm{~V}$. Redistribution, on the right, shows $v_{\mathrm{sc}}$ starting at $t_{\mathrm{open}}=0 \mathrm{~s}$ when $i_{\mathrm{sc}}$ is first disconnected. 


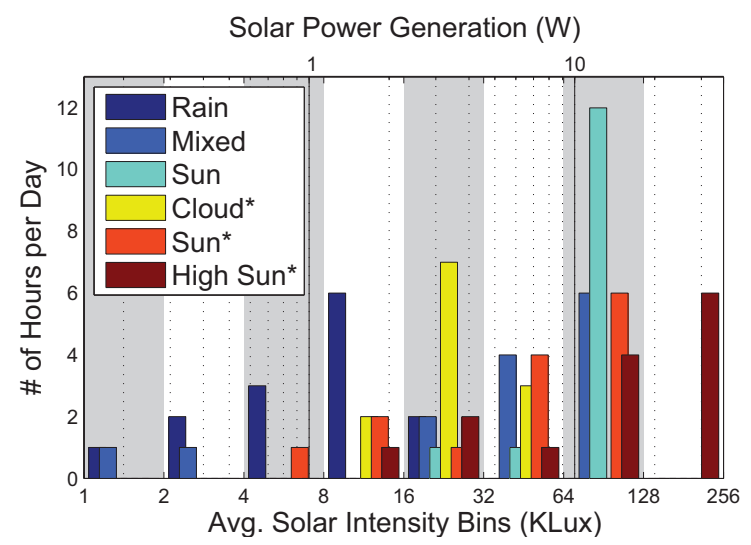

Figure 3: The histogram of solar generation from 21 RadioShack $1.5 \mathrm{~W}$ panels (max. $31.5 \mathrm{~W}$ at $200 \mathrm{KLux}$ ) varies by two orders-of-magnitude, resulting in significant potential for non-ideal supercapacitor behavior while buffering the energy. The marked data $(*)$ is recorded in Italy [35], whereas the rest of the days are measured in Rochester NY, USA [10]. 


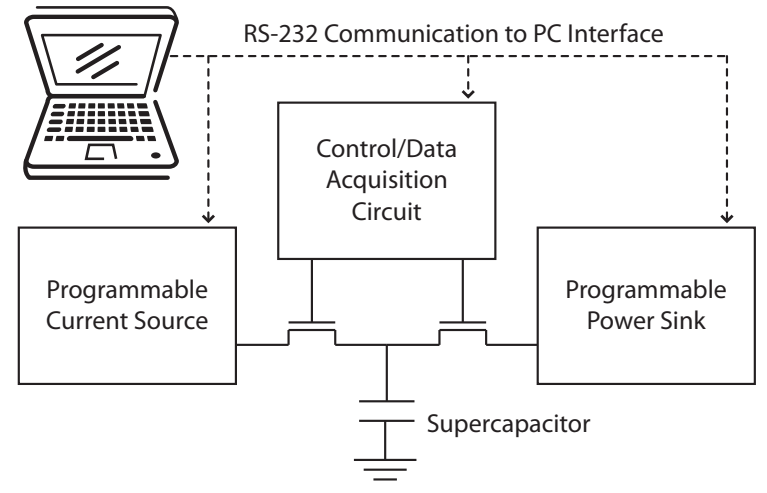

(a)

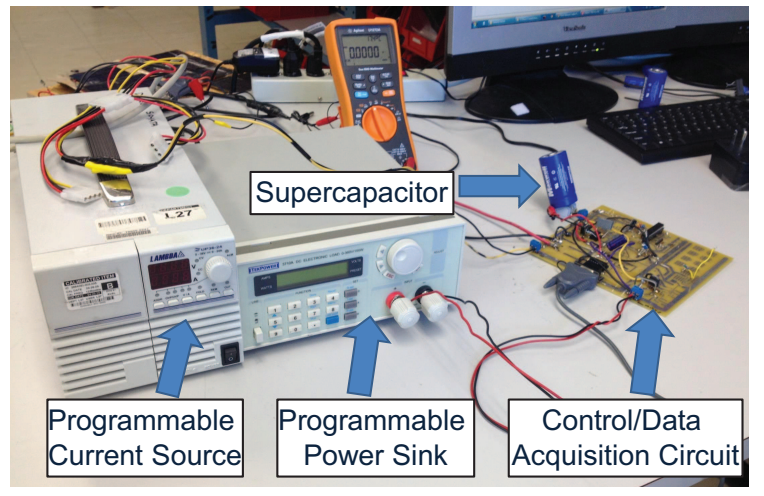

(b)

Figure 4: The validation setup depicted in (a) uses a PIC microcontroller to control the charging and discharging currents via MOSFET gates. Data logging and high level control of each module is done from a workstation running Matlab via RS-232 serial connections. The corresponding arrangement of the test equipment is pictured in (b), showing a $350 \mathrm{~F}$ supercapacitor. 


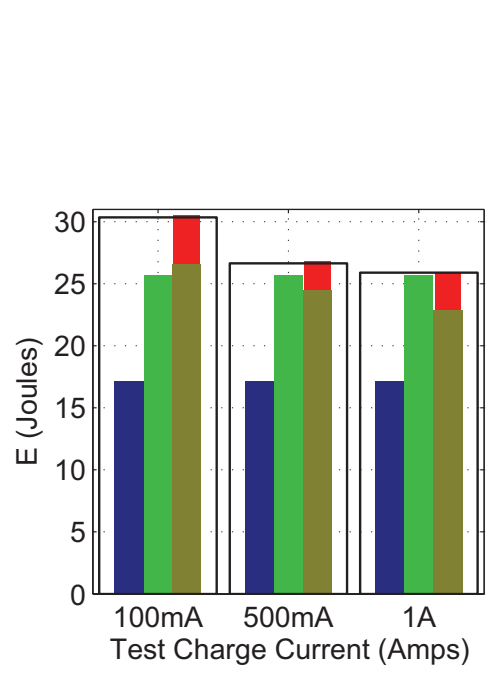

(a) $5 \mathrm{~F}$
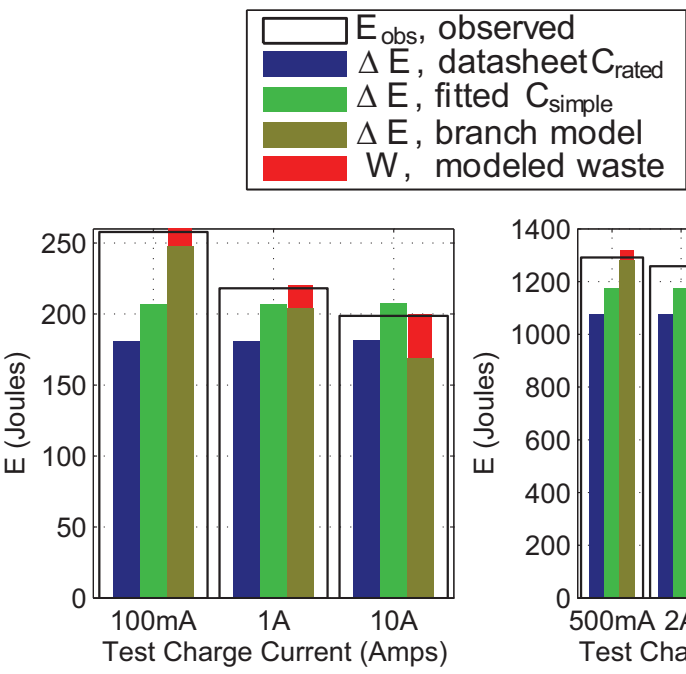

(b) $50 \mathrm{~F}$

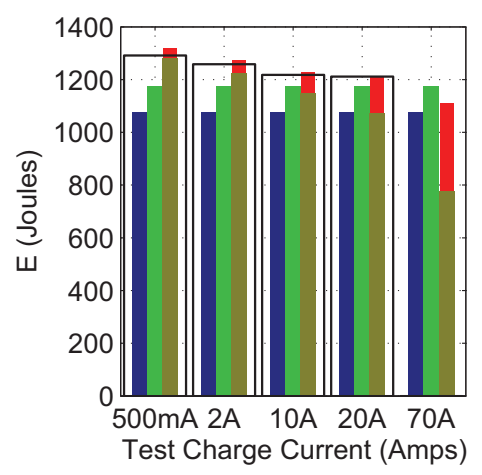

(c) $350 \mathrm{~F}$

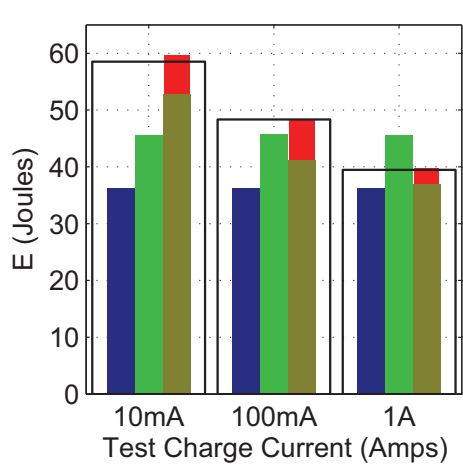

(d) $10 \mathrm{~F}$

Figure 5: The energy ( $E_{\mathrm{obs}}$, observed) that is required to charge the supercapacitor from rest to its rated voltage depends on the charging current. Three techniques for predicting $E_{\text {obs }}$ are compared. The first two techniques, (datasheet $C_{\text {rated }}$ ) and (fitted $C_{\text {simple }}$ ) used a fixed capacitance. Alternatively, the three branch equivalent circuit (branch model) is used to predict both the stored energy, $\Delta E$, and internal losses, $W$. Results for the Maxwell supercapacitors are shown in (a), (b), and (c), while (d) shows the results for the Illinois Capacitor device. 


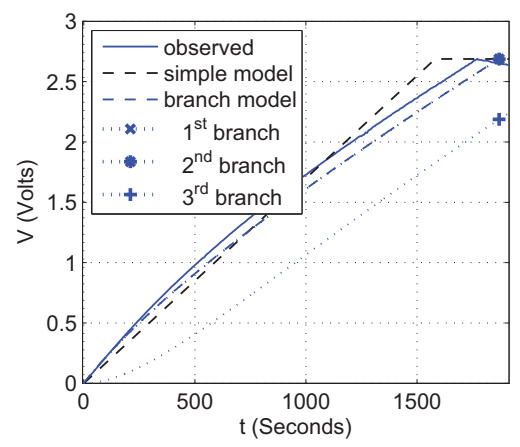

(a) $100 \mathrm{~mA}$ charging.

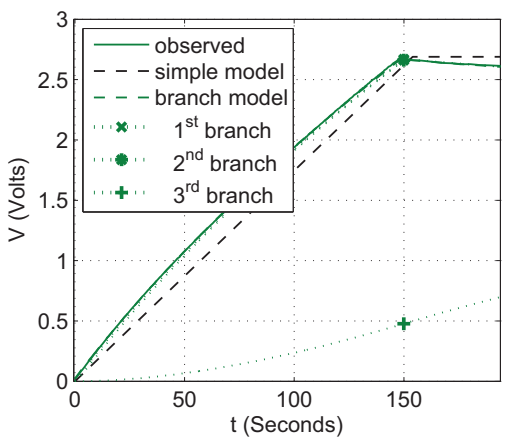

(b) $1 \mathrm{~A}$ charging.

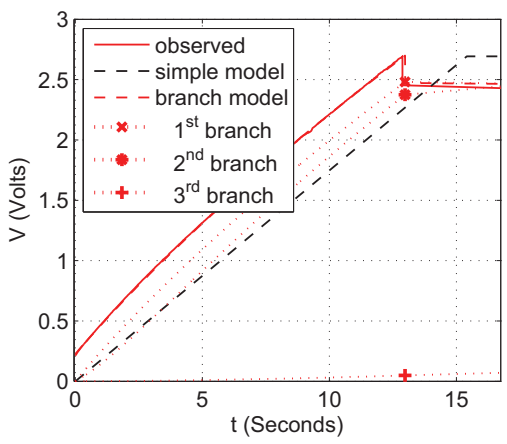

(c) $10 \mathrm{~A}$ charging.

Figure 6: These $100 \mathrm{~mA}, 1 \mathrm{~A}$ and $10 \mathrm{~A}$ charging tests for the $50 \mathrm{~F}$ supercapacitor in (a), (b), and (c), provide the (observed) data used to fit the parameters. The resulting parameters for the three branch equivalent circuit (branch model) and simple capacitive (simple model) are then simulated in order to predict the duration and energy that are required to charge the supercapacitor from a completely discharged state to its rated voltage $(2.7 \mathrm{~V})$ at each current. 


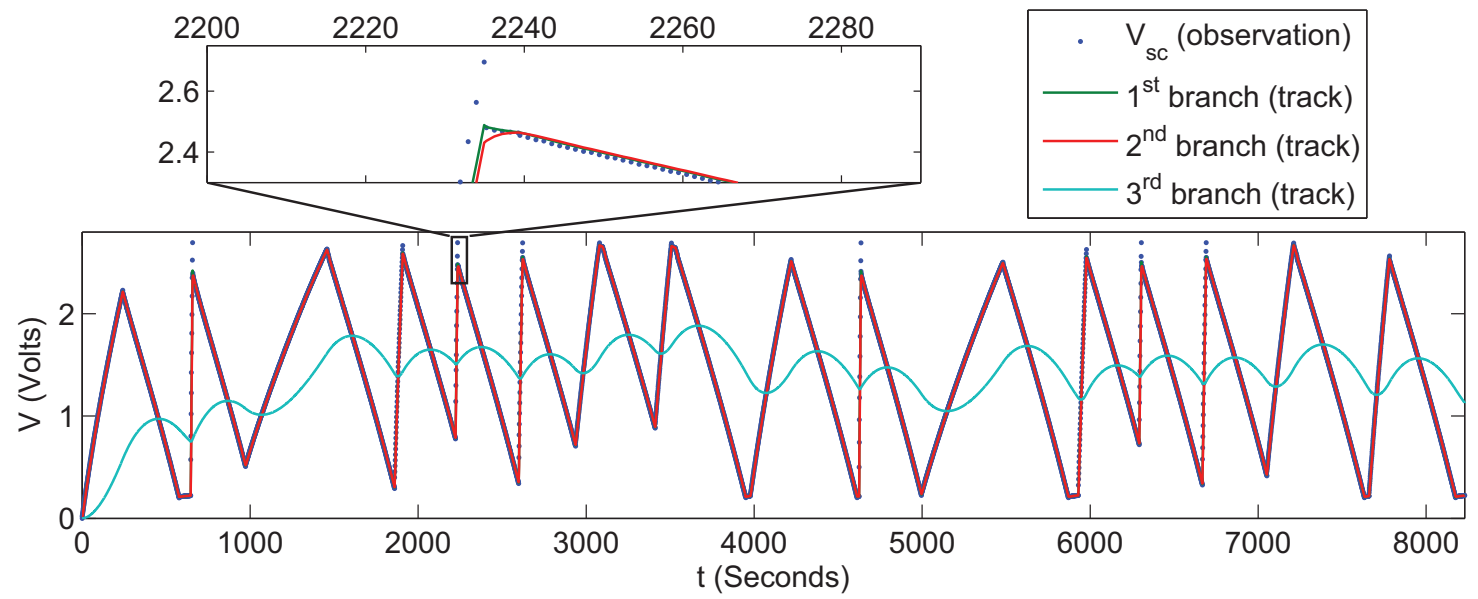

Figure 7: The proposed Kalman technique for tracking the 50F supercapacitor's internal SOC is experimentally validated over a series of various charging currents designed to show the range of possible solar variability from Fig. 3 . The top, zoomed, axes shows the much shorter time scale of the $1^{\text {st }}$ and $2^{\text {nd }}$ branch voltages. 


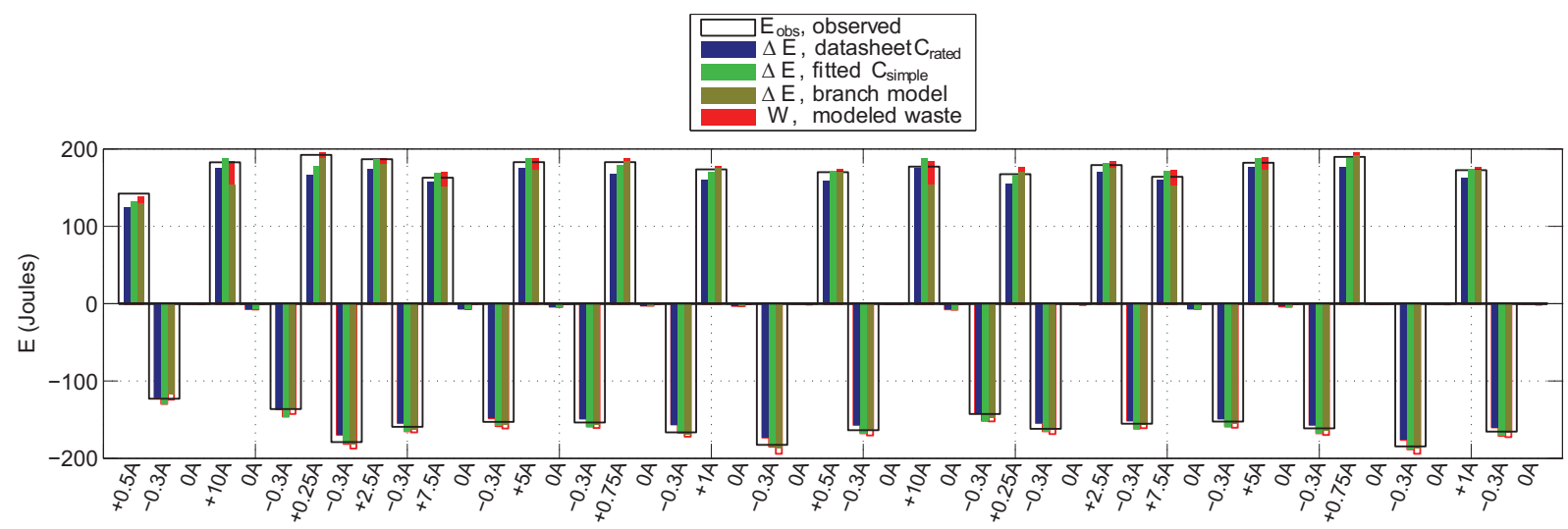

Figure 8: For each charging $(>0 \mathrm{~A})$, redistribution $(0 \mathrm{~A})$, and discharging $(<0 \mathrm{~A})$ duration of the tracking experiment shown in Fig. 7, the energy transfer, $E_{\text {obs }}$ (observed), is predicted using both models: the (branch model) and the fixed capacitive models using $C_{\text {rated }}$ (datasheet) from the manufacturer, and $C_{\text {simple }}$ (fitted) from the training data. The RMS (root mean squared) error is 3.8J for the branch model, compared to 5.5 $\mathrm{J}$ and $8.3 \mathrm{~J}$ for the respective fitted and datasheet fixed capacitive models. Corresponding numeric data is given in the supplementary material in Table S2. 
Table 1: Parameter matrices for three branch equivalent circuit differential equation system in (16) and (17).

Table 2: The proposed (LMSE fit) technique is compared to prior work (ref. [15] fit) for estimating the parameters of the three branch equivalent circuit. The parameters are estimated using simulated measurement data for a 470F DLC with (true) parameters specified in [15].

Table 3: The propose LMSE technique is used to estimate the parameters of the $5 \mathrm{~F}, 10 \mathrm{~F}, 50 \mathrm{~F}$, and $350 \mathrm{~F}$ supercapacitors using observed training data.

Figure 1: As shown in (a), each of a supercapacitor's terminals is connected to a porous electrode material such as activated carbon. The conductive electrodes are immersed in an electrolyte but separated by a membrane which only allows the ions in the electrolyte to pass through. Diffusion of the ions into the porous surface is modeled by the three branch equivalent circuit [15], shown in (b). Each branch has a time constant of increasing duration, $R_{\text {serial }} C_{1}<R_{2} C_{2}<R_{3} C_{3}$, modeling initial, intermediate, and long term behavior.

Figure 2: Charging, on the left, shows the terminal voltage of a 50F Maxwell Boostcap Ultracapacitor as it is charged with $100 \mathrm{~mA}, 1 \mathrm{~A}$, and $10 \mathrm{~A}$ constant currents. Because Charging is scaled to Coulombs, $q$, it is easy to see that a supercapacitor does not strictly follow the proportion, $v_{\mathrm{sc}}=1 / \mathrm{C} \cdot \mathrm{q}$, predicted by the simple model. The supercapacitor always starts in a completely discharged state, and current, $\mathrm{i}_{\mathrm{sc}}$, is disconnected when $\mathrm{v}_{\mathrm{sc}}$ reaches the device's rated voltage, 2.7V. Redistribution, on the right, shows $\mathrm{v}_{\mathrm{sc}}$ starting at $\mathrm{t}_{\mathrm{open}}=0 \mathrm{~s}$ when $\mathrm{i}_{\mathrm{sc}}$ is first disconnected.

Figure 3: The histogram of solar generation from 21 RadioShack 1.5W panels (max. 31.5W at 200KLux) varies by two orders-of-magnitude, resulting in significant potential for non-ideal supercapacitor behavior while buffering the energy. The marked data $\left({ }^{*}\right)$ is recorded in Italy [35], whereas the rest of the days are measured in Rochester NY, USA [10].

Figure 4: The validation setup depicted in (a) uses a PIC microcontroller to control the charging and discharging currents via MOSFET gates. Data logging and high level control of each module is done from a workstation running Matlab via RS-232 serial connections. The corresponding arrangement of the test equipment is pictured in (b), showing a 350F supercapacitor.

Figure 5: The energy ( $E_{o b s}$, observed) that is required to charge the supercapacitor from rest to its rated voltage depends on the charging current. Three techniques for predicting $\mathrm{E}_{\mathrm{obs}}$ are compared. The first two techniques, (datasheet $C_{\text {rated }}$ ) and (fitted $C_{\text {simple }}$ ) used a fixed capacitance. Alternatively, the three 
branch equivalent circuit (branch model) is used to predict both the stored energy, $\Delta \mathrm{E}$, and internal losses, W. Results for the Maxwell supercapacitors are shown in (a), (b), and (c), while (d) shows the results for the Illinois Capacitor device.

Figure 6: These 100mA, 1A and 10A charging tests for the 50F supercapacitor in (a), (b), and (c), provide the (observed) data used to fit the parameters. The resulting parameters for the three branch equivalent circuit (branch model) and simple capacitive (simple model) are then simulated in order to predict the duration and energy that are required to charge the supercapacitor from a completely discharged state to its rated voltage (2.7V) at each current.

Figure 7: The proposed Kalman technique for tracking the 5OF supercapacitor's internal SOC is experimentally validated over a series of various charging currents designed to show the range of possible solar variability from Fig. 3 . The top, zoomed, axes shows the much shorter time scale of the $1^{\text {st }}$ and $2^{\text {nd }}$ branch voltages.

Figure 8: For each charging $(>0 A)$, redistribution $(O A)$, and discharging $(\angle O A)$ duration of the tracking experiment shown in Fig. 7, the energy transfer, $E_{\text {obs }}$ (observed), is predicted using both models: the (branch model) and the fixed capacitive models using $\mathrm{C}_{\text {rated }}$ (datasheet) from the manufacturer, and $\mathrm{C}_{\text {simple }}$ (fitted) from the training data. The RMS (root mean squared) error is 3.8J for the branch model, compared to $5.5 \mathrm{~J}$ and $8.3 \mathrm{~J}$ for the respective fitted and datasheet fixed capacitive models.

Corresponding numeric data is given in the supplementary material in Table S2. 